\title{
EDITORIAL
}

\section{Welcome to Frontiers of Materials Science in China}

Frontiers of Materials Science in China (FMSC) is now three years old. Thanks to the excellent work of its founding editorial board headed by Academician Chun-Li BAI, FMSC has grown steadily and about 180 original papers have been published. FMSC papers have been cited by other journals including those with an impact factor higher than 6 . Non-Chinese papers have also increased gradually. The current editorial board reaffirms FMSC' dedication to publishing papers of frontier studies of all materials fields from China and abroad; FMSC aspires to become one of the most read journals in the materials community.

Of particular interest to FMSC are papers on new materials that enable applications in information, energy and medicine as well as in the biological and environmental sciences. On these topics nano materials will be a focus.

Also of continued interest are lessons learned from nature as they apply to material development. Understanding biomineralization can inform biomimetic synthesis for tailored applications. Understanding cell behavior and its underlying processes can guide designs of biomaterials and smart materials.

Performance evaluation of materials will benefit from physically based modeling that is reproducible and robust. Modeling papers strengthened by the inclusion of performance data will be of interest.

Lastly, reviews of the above topics and, more broadly, new advances in various branches of materials science will be welcome.

Reflecting on the above discussion, the following is a revised list of topics that comprise the scope of FMSC. From this list submitting authors will be asked to identify the topics that are dealt with by their work.

- Frontier materials synthesis and characterization

- Materials performance

- Materials applications in energy, information and biotechnology

- Novel methods for materials manufacturing

- Materials modeling

- Nano materials

- Biomaterials including biomimetics and biomineralization

- Advanced ceramics

- Polymers and composites

- New metallic materials

Please let us hear from you regarding any suggestions to our ongoing process of improving FMSC.

\section{Editor-in-Chief}

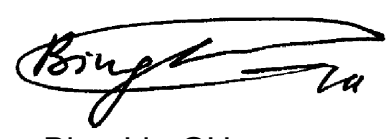

Bing-Lin GU

\section{Associate Editors-in-Chief}

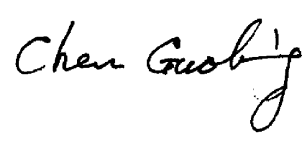

Guo-Liang CHEN,

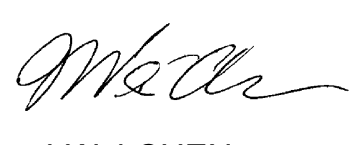

I-Wei CHEN,

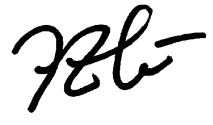

Fu-Zhai CUI,

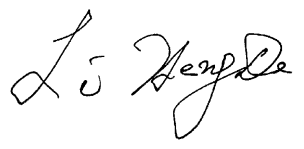

Heng-De LI,

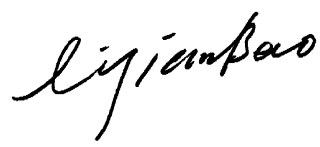

Jian-Bao LI 